Tohoku J. exp. Med., 1986, 149; 407-416

\title{
Sex Determination of the Human Skull Based upon Line Drawing from Roentgen Cephalograms
}

\author{
Masato Funayama, Yasuhiro Aoki, Toshiyuki Kudo \\ and KaOru Sagisaka \\ Department of Forensic Medicine, Tohoku University School \\ of Medicine, Sendai 980
}

\begin{abstract}
Funayama, M., Aoki, Y., Kudo, T. and Sagisaka, K. Sex Determination of the Human Skull Based upon Line Drawing from Roentgen Cephalograms. Tohoku J. exp. Med., 1986, 149(4), 407-416 — The shape of the human skulls was studied with the aid of a personal computer to establish sex differences in quantitative anatomical terms. On the roentgen cephalograms from 50 adult males and 50 adult females, the lateral profile was transcribed onto an acetate sheet, on which the contour from the nasal apex to the forehead was digitized on a tablet digitizer into a series of dots which were input into the computer system. After this chain of dots was simulated by a spline function, the places most typically reflecting sex difference were determined in the profile based on the radius of curvature computed at each dot. The eminence of glabella and the nasal root, shown to be the places of skull apparently most characteristic of sex, were approximated to circular arcs with the least squares method, the radii of which were expected to serve for sexing. In both places, Student's $t$-test revealed a significant difference between the male and female groups $(p<0.01)$. It was thus demonstrated that in male the eminence of glabella and the nasal concavity develop much more markedly than in female, presenting as a clear skeletal difference between both sexes. - sex determination; human skull ; roentgen cephalogram
\end{abstract}

So far, two different methods have been used in sex determination from human skeleton; the first is the metric analysis comprising more than one measurement of bone, and the second is the conventional visual approach where sexing relies mainly on rather intuitive recognition of some gross morphological features of skeleton. In the former, measurements are performed according to the method of Martin (1914) or that of Martin and Saller (1957). However, these methods have hitherto been reported to produce overlapping between male and female to such various extents that recently, several studies have resorted to the application of the multivariate discriminant function first introduced by Fisher (1936) in taxonomic studies. Also the discriminant scores for the Japanese were calculated on the skull, pelvis, scapula and bones of extremities (Hanihara 1958, 1959, 1981 ; Hanihara et al. 1964 ; Akamine 1960 ; Kimura 1971; Tanaka et al.

Received February 1, 1986; accepted for publication June 24, 1986. 
1979). However, there is a problem inherent in this technique; measurements are required to perform at a large number of sites to lower the probability of misclassification, which is virtually impossible in dealing with partially broken bones, a very frequent experience in forensic medicine. Thus, the judgment in actual practice almost entirely is performed by the conventional visual observation, where much experience and skill are still required in examining bones of unknown sex. In this report, we describe our attempt at establishing, in quantitative anatomical terms, the sites of crania suitable for sex determination.

The roentgen cephalogram was introduced into orthodontic studies by Broadbent (1931) to visualize the growth patterns of maxillofacial skeleton and its change. Since then, this method has been widely used not only in the prediction of skeletal growth (Broadbent 1937; Nanda 1955) but in the diagnosis, treatment and research of malocclusion (Ricketts 1957 ; Sassouni 1958; Graber 1968). In the present analysis, the lateral profiles of crania in cephalograms were transcribed on acetate sheets by tracing, and the places available for sexing were determinated by inputting the profile patterns into a personal computer.

\section{Material and Methods}

\section{Examined cases}

The basic material was the roentgen cephalograms of young adults offered by the Department of Orthodontics, School of Dentistry, Tohoku University. These were taken on candidates, mainly students of the Dental Technician School and those of the Dental School of this university. Cephalograms from fifty males and those from fifty females were chosen at random. The age of the subjects ranged from 18 to 31 years (Table 1). The distance from the subject to the $\mathrm{x}$-ray tube was fixed at $235 \mathrm{~cm}$, that to the film at $150 \mathrm{~mm}$.

\section{Analytical method}

The places for graphic analysis were set in the lateral profile so as to satisfy the following three requirements ; 1) the sites reported to show clear sex difference, 2) the sites with smooth outline and therefore easy for graphic processing and 3) the sites hardly broken or influenced by aging. In a review of the lateral profile from the nasal apex to the forehead in the cephalograms, the eminence of glabella and the nasal root were picked up as places satisfying the above requirements, and each of these contours was approximated

TABLE 1. Age distribution of the cases

\begin{tabular}{ccc}
\hline Age & Male & Female \\
\hline $18 \sim 19$ & & 26 \\
$20 \sim 21$ & 12 & 10 \\
$22 \sim 23$ & 16 & 7 \\
$24 \sim 25$ & 12 & 5 \\
$26 \sim 27$ & 8 & 1 \\
$28 \sim 29$ & 1 & \\
$30 \sim 31$ & 1 & 1 \\
\hline Total & 50 & 50 \\
\hline
\end{tabular}


to a circular arc.

In practice, the contour in each cephalogram was transcribed onto an acetate sheet by tracing with a pencil. The superciliary arch that sometimes appeared as a thin contour above the glabella was ignored, and the thick contour of the eminence of glabella was drawn. A drawing was placed on a tablet digitizer (Bit Pit One, MUTOU, Tokyo) on which a sheet of section paper had been attached, and the profile was input using a four-button cursor at a point mode with such a velocity that a dot might be generated at about every $0.5 \mathrm{~mm}$ of contour length; of course, this implies to transform the profile into a chain of dots, the coordinates of which are serially transferred into a computer system. The error in coordinate values read on the tablet was less than $0.1 \mathrm{~mm}$. The computer system comprised a 16-bit NEC personal computer (PC-9801E, NEC, Tokyo), a color character display (PCKD551K, NEC, Tokyo), a floppy disk unit (LFD-880, Tokyo Densikagaku kizai Co., Tokyo) and a serial printer (PC-PR201, NEC, Tokyo).

In practice, the places in question such as the eminence of glabella or nasal root have to be defined in unambiguous terms before analyzing the morphological features, but there is no anatomical landmark to pinpoint accurately these places so long as one relies on conventional visual recognition. For graphic processing, however, it is desirable to point these places on strict quantitative-geometric basis. In this view, the sequence of dots input were submitted to computational approximation to a cubic spline function (Ahlberg et al. 1967 ; Ichida and Yoshimoto 1985). It was assumed that the radius of curvature becomes the largest at the transferential point of a curve (Fig. 1). On this basis, the radius of curvature was computed at each of a chain of dots, to which the lateral profile was transformed. Finally, three dots with the comparatively large radius of curvature were selected, partially at user's own will, as representing the "peak" points and therefore corresponding to the specific landmarks. These "peak" dots usually occurred on the contour of nasal bone (A), near Nasion (B), and near the upper end of the glabella (C), and the two fractions of the counter $\mathrm{A}-\mathrm{B}$ and $\mathrm{B}-\mathrm{C}$ were defined to be the characteristic places. In our study, the ranges $\mathrm{B}-\mathrm{C}$ and $\mathrm{A}-\mathrm{B}$ were regarded as corresponding to the eminence of glabella and the nasal root, respectively. In the cubic spline function, on which basis the radius of curvature at each dot was computed, fragments of many polynomials are joined to form a single continuous curve, so that its first and second derivatives (Fig. 2) are continuous, ensuring the smoothness throughout the region assigned.

In applying the cubic spline, trials were repeated to generate the knots, where two separate polynomials are joined, until a certain level of fitting to the actual profile was attained; whether a desired fitting had been satisfied was judged according to the "Powell's trend" (Powell 1970 ; Ichida and Yoshimoto 1985). Initially, approximation was tried with only three knots, the both ends and the middle point in a series of dots, followed by an iterative procedure. On each iteration, the degree of fitting of the assumed spline to the actual chain of dots were tested by the least squares method, and then the residuals were tested for the trend of Powell.

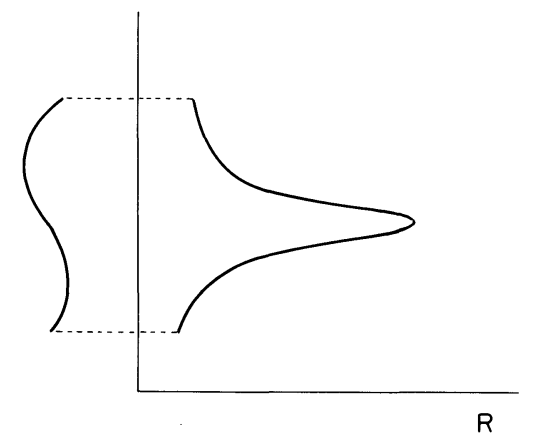

Fig. 1. The change of the radius of curvature ( $R$, right) assumed for a profile exhibited in the left. 


$$
\begin{array}{rlr}
S(x)= & M_{i-1} \frac{\left(x_{i}-x\right)^{3}}{6 h_{i}}+M_{i} \frac{\left(x-x_{i-1}\right)^{3}}{6 h_{i}} & \\
& +\left(y_{i-1}-\frac{M_{i-1} h_{i}^{2}}{6}\right) \frac{x_{i}-x}{h_{i}}+\left(y_{i}-\frac{M_{i} h_{i}^{2}}{6}\right) \frac{x-x_{i-1}}{h_{i}} & \\
S^{\prime}(x)= & & (i=1,2, \cdots, n) \\
& -\frac{M-M_{i-1} \frac{\left(x_{i}-x\right)^{2}}{2 h_{i}}+M_{i} \frac{\left(x-x_{i-1}\right)^{2}}{2 h_{i}}+\frac{y_{i}-y_{i-1}}{h_{i}}}{6} h_{i} & \\
S^{\prime \prime}(x)= & & \\
M_{i-1} \frac{x_{i}-x}{h_{i}}+M_{i} \frac{x-x_{i-1}}{h_{i}} & (i=1,2, \cdots, n) \\
& & (i=1,2, \cdots, n) \\
\text { were } M_{i}=S^{\prime \prime}\left(x_{i}\right) & (i=1,2, \cdots, n) \\
h i=x_{i}-x_{i-1} & (i=1,2, \cdots, n)
\end{array}
$$

Fig. 2. The cubic spline function (Ahlberg et al. 1967).

The "Powell's trend" examines whether there is a trend between two knots, depending on the values of the residuals $\left(r_{k}\right)$

$$
r_{k}=S\left(X_{k}\right)-F_{k} \quad(k=p, p+1, \ldots, q)
$$

where $F_{k}$ is the actual value of interval, and $S\left(X_{k}\right)$ is an approximate function, i.e., a cubic spline. The condition of a definite trend is

$$
T=\sum_{k=p}^{q} r_{k-1} r_{k}<\sum_{k=p+1}^{q} r_{k}^{2} / \sqrt{q-p}=U
$$

However, when applied to actual data, the number of approximate functions because considerably large with a lot of knots generated, and this condition proved to be too strict

Step 1. Smoothing of data (landmarks detection)

Cephalometric tracing

$$
\downarrow
$$

Transformation of the lateral profile into a chain of dots through a tablet digitizer

Curve simulation of the chain of dots by a spline function

Calculation of the radius of curvature on each dot

Determination of the landmarks out of the "peak" dots

Step 2. Metric analysis at the characteristic places

Choice of a characteristic place between the landmarks

Substitution of initial condition

$$
\downarrow
$$

Iterative procedures of circular arc fitting with the least squares method

Output of the radius of circular arc

Fig. 3. The two steps for analyzing the characteristic places. 
Sex Determination of Human Skull

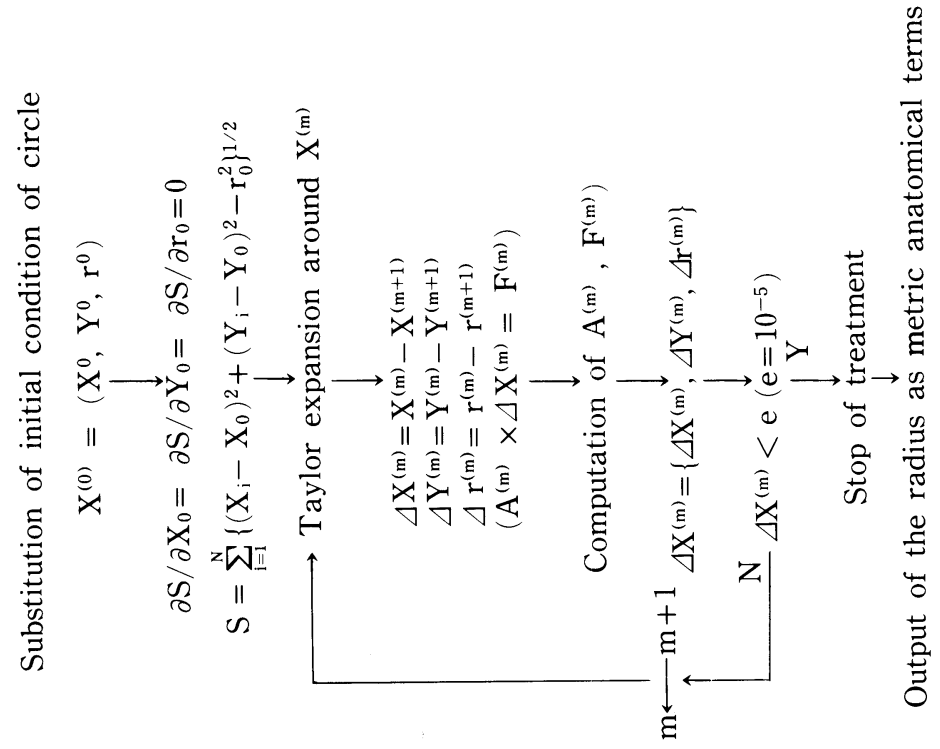

常

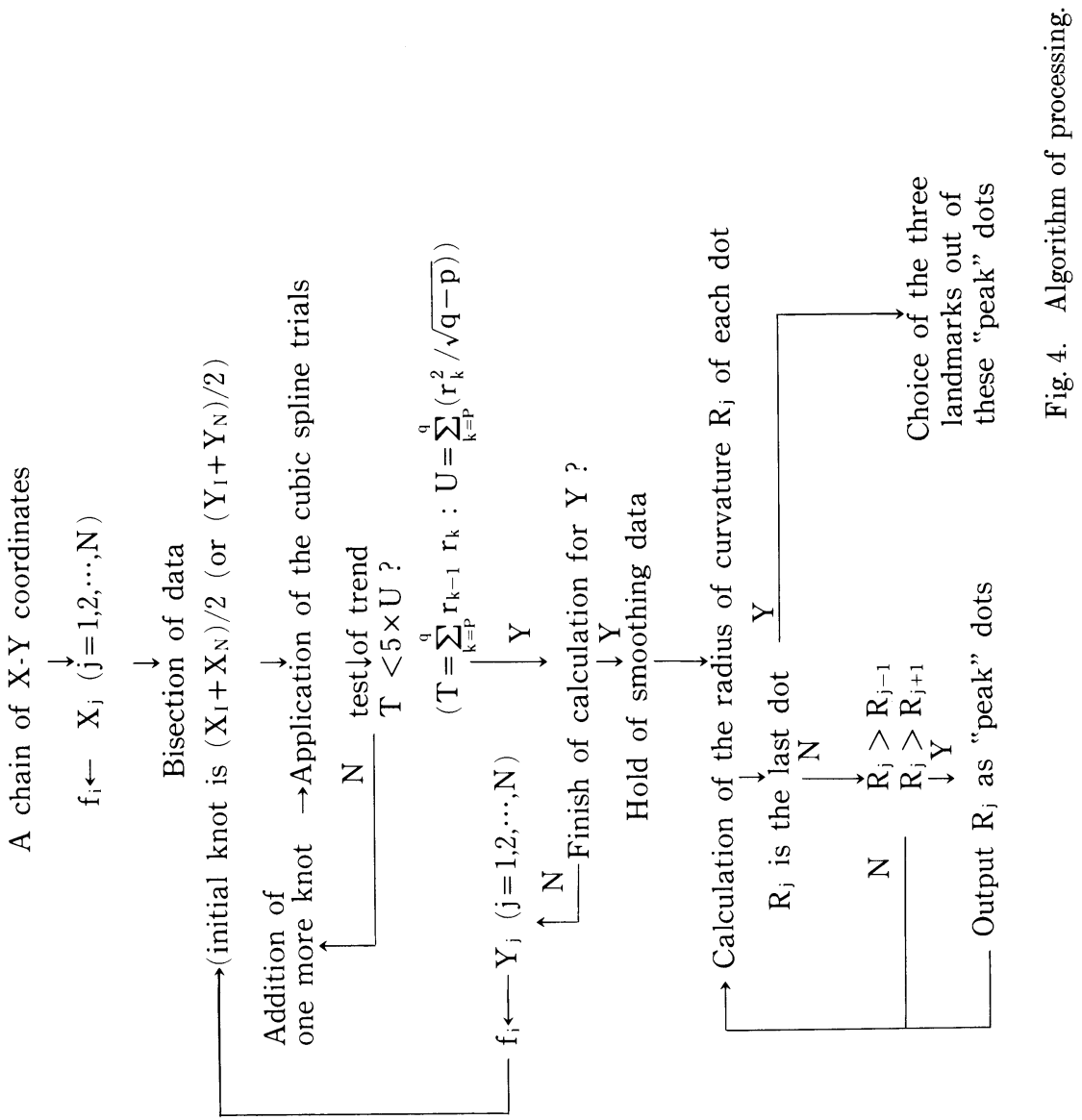



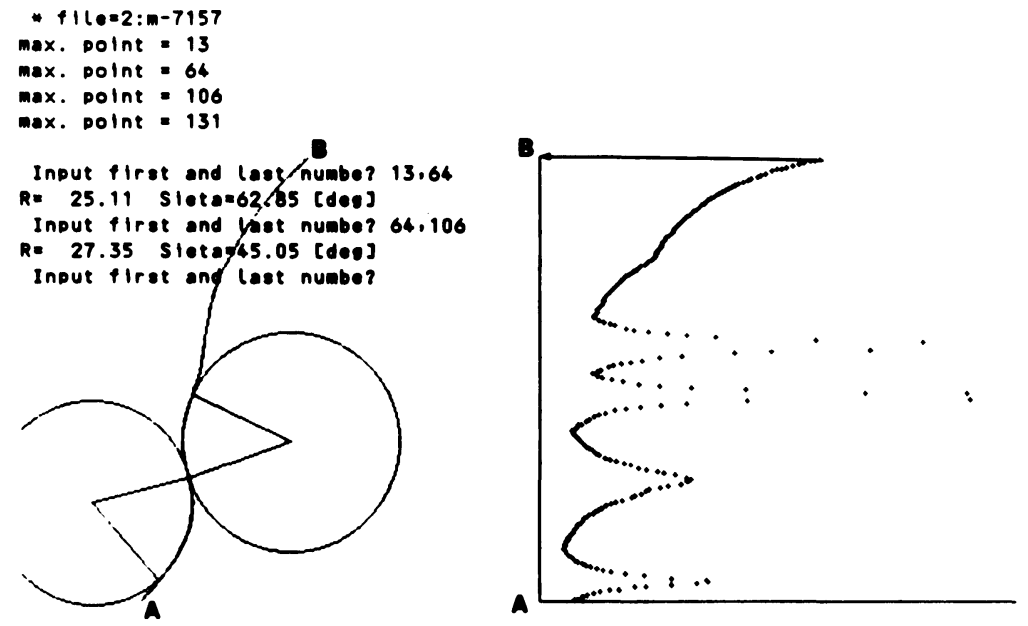

Fig. 5. The output on CRT. The ordinate in the right shows a chain of dots digitized from the nasal apex (A) to the upper part of the forehead (B) in the profile shown in the left, and the abscissa shows the radius of curvature at each dot. This case displays four "peak" dots (top left), and the range (13)-(64) is regarded as the nasal root an (64)-(106) the eminence of glabella.

to define the landmarks. Accordingly, we relaxed the restriction into:

$$
T<U \times 5
$$

We assumed that there was a trend if the condition was satisfied. This appeared to serve as a criterion in defining the characteristic places in a long series of dots.

Each pair of characteristic places selected by the above landmarks were approximated to a circular arc with the least squares method, and the feature of a given cranium was defined by the radius of the arc. The formula to calculate the radius is

$$
r=\left\{\left(x-x_{0}\right)^{2}+\left(y-y_{0}\right)^{2}\right\}^{1 / 2}
$$

where $r$ is the radius of the approximated circle derived from a series of coordinates $\left(x_{i}, y_{i}\right)$ $(i=1,2, \ldots, N)$. The residual sum of squares, $S$, is defined as

$$
\begin{aligned}
S & =\sum_{i=1}^{N}\left\{r\left(x_{i}, y_{i}\right)^{2}-r^{2}\right\}^{1 / 2} \\
& =\sum_{i=1}^{N}\left\{\left(x_{i}-x\right)^{2}+\left(y_{i}-y\right)^{2}-r^{2}\right\}^{1 / 2}
\end{aligned}
$$

Using the Gauss-Newton system (Nakagawa and Koyanagi 1984), the parameters ( $x, y, r$ ) to minimize $S$ were determined. The initial value was given by the circle passing the three points ; the terminal and the median points of a characteristic place. Moreover, the central angle of each circular arc was calculated to compare the error arising during several trials.

These processes can be divided into two steps. The first is the smoothing of data to determine the landmarks and the second is the calculation of the radius at the characteristic places, that are defined as fractions of the profile inserted between the landmarks. Fig. 3 outlines these two processes, and Fig. 4 the algorithms. The format of the final output on the CRT is shown in Fig. 5 as an example.

\section{Statistical analysis of feature}

The values of radii obtained from the measurements on the eminence of glabella and the nasal root were submitted to basal statistical treatment, and the significance of the difference 
TABLE 2. The difference in the radius of curvature $(\mathrm{mm})$ estimated in a part of trial for 10 cases selected arbitrarily

\begin{tabular}{|c|c|c|c|c|c|c|c|}
\hline \multirow{2}{*}{ Sex } & \multirow{2}{*}{ Case No. } & \multicolumn{3}{|c|}{ The eminence of glabella (mm) } & \multicolumn{3}{|c|}{ The nasal root (mm) } \\
\hline & & 1st trial & 2nd trial & $|\Delta|$ & 1st trial & 2nd trial & $\Delta \mid$ \\
\hline \multirow{5}{*}{ Male } & 6 & 16.9 & 18.0 & 1.1 & 14.3 & 12.7 & 1.6 \\
\hline & 69 & 25.6 & 21.6 & 4.0 & 23.4 & 28.2 & 4.8 \\
\hline & 174 & 26.1 & 22.5 & 3.6 & 11.3 & 11.7 & 0.4 \\
\hline & 200 & 18.6 & 18.0 & 0.6 & 12.9 & 14.5 & 1.6 \\
\hline & 209 & 20.5 & 20.3 & 0.2 & 19.4 & 19.4 & 0.0 \\
\hline \multirow{5}{*}{ Female } & 37 & 37.9 & 37.6 & 0.3 & 20.6 & 18.7 & 1.9 \\
\hline & 134 & 39.6 & 42.9 & 3.3 & 29.1 & 27.1 & 2.0 \\
\hline & 177 & 49.5 & 51.1 & 1.6 & 19.2 & 18.3 & 0.9 \\
\hline & 179 & 37.2 & 36.6 & 0.6 & 31.6 & 26.2 & 5.4 \\
\hline & 361 & 40.0 & 35.5 & 4.5 & 23.1 & 23.7 & 0.6 \\
\hline
\end{tabular}

TABLE 3. The statistical treatment of the results for the eminence of glabella and the nasal root

\begin{tabular}{lcccrrrr}
\hline Variable & Sex & No. & $\begin{array}{c}\text { Mean } \\
(\mathrm{mm})\end{array}$ & $\begin{array}{c}\text { S.D. } \\
(\mathrm{mm})\end{array}$ & $\begin{array}{c}F \\
\text { value }\end{array}$ & $\begin{array}{c}t \\
\text { value }\end{array}$ & $\begin{array}{c}\text { Level of } \\
\text { significance }\end{array}$ \\
\hline \multirow{2}{*}{ The eminence of glabella } & M & 50 & 22.09 & 4.21 & 7.67 & 10.20 & $<0.01$ \\
& F & 46 & 40.65 & 11.66 & & & \\
\hline \multirow{2}{*}{ The nasal root } & M & 50 & 17.49 & 4.74 & 2.08 & 4.90 & $<0.01$ \\
& F & 50 & 23.26 & 6.84 & & & \\
\hline
\end{tabular}

S.D., standard deviation.

between the means of the male and the female groups was examined by the Student's $t$-test. Four female cases with missing values on the eminence of glabella were excluded from the analysis.

\section{Study of error}

Errors were considered to arise in the process of data input and computation. So, the following two tests were performed. In the first place, a circular arc of known radius was input, and the difference between the real and the computed values for the radius was examined. In the present study, we made it a rule to exclude a case if an error over $10 \%$ was generated. On the other hand, measurements were repeated to know how variously a radius can be computed when a circular arc was digitized along different central angles, i.e., generating different number of dots. In the next place, each five cases were randomly chosen from the male and female groups, in which a series of data processing was tried all over again. The difference in the results between the first and second trials was examined.

\section{Results}

\section{Appraisal of the Technical and Instrumental Errors}

Cases were excluded as missing values when, as mentioned above in the treatment of errors, the central angle of circular arc proved to be less than 20 
degrees or the number of component dots was less than 30 , because an error over $10 \%$ was expected to ensue. The computation of the coordinate values corresponding to the eminence of glabella revealed that there were four cases in the female group with a central angle less than 20 degrees, and these were regarded as missing values on statistics. Repeated trials of input and computation on the eminence of glabella and the nasal root produced a variation of radius ranging from 0.2 to $4.5 \mathrm{~mm}$ for the former, and 0 to $5.4 \mathrm{~mm}$ for the latter place, respectively (Table 2).

\section{Values Specific of Sex}

The descriptive statistics for the reference places are shown in Table 3 together with the results of $t$-test between the male and female groups. The mean radius of circular arc corresponding to the eminence of glabella on cephalometric tracing was $22.1 \mathrm{~mm}$ in males and $40.7 \mathrm{~mm}$ in females, that to the nasal root 17.5 $\mathrm{mm}$ in males and $23.3 \mathrm{~mm}$ in females, respectively. The difference was demonstrated to be significant $(p<0.01)$ at both places, with evidently smaller radius in the male than in the female group. The standard deviation of radii was 4.21 in males and 11.66 in females at the eminence of glabella, and 4.74 in males and 6.84 in females at the nasal root. It may be induced from this result that if judgment of sex were performed on these distributions alone, one male and 5 females of our series would have misclassified by the measurement at the eminence of glabella, and fifteen males and seventeen females at the nasal root.

\section{Discussion}

The age of the subjects examined in this study were in the twenties except for the eight females aged eighteen and eighteen females aged nineteen. It was shown in the study of Asai (1973) that the maxillofacial skeleton, completing its growth by the age of seventeen years in females, shows no significant anatomical change on roentgen cephalograms in the following years. In view of this, the above 26 females under twenty may safely be included, presuming that they have skeletons sufficiently grown up with regard to the clavaria as well the maxillofacial bones.

Although it has been pointed out that the glabella and the nasal root are places of the skull typically visualizing sex difference, objective discrimination of sex on these places has required experience and skill. The present study, with the aid of a personal computer, clearly demonstrated an anatomical sex difference in the lateral profile from the forehead to the nasal apex. Roentgen cephalograms were submitted to tracing of the skeletal profile which was digitized and input into a computer. The radii of circular arc at these places were computed, demonstrating a significantly smaller radius in males than in females $(p<0.01)$. The difference was especially clear at the eminence of glabella where sex could be determined with an accuracy of more than $90 \%$. In the previous studies using 
discriminant function, though combining measurements at many places of the skull, the range of correct identification was reported to be lower than $90 \%$; 83.1$86.4 \%$ by Hanihara (1959) and $86.1-87.3 \%$ by Tanaka et al. (1979) on the Japanese, and $84.0-86.4 \%$ by Giles and Elliot (1963) on the negro and white races. The method we have applied to sex determination of human skull thus appear more effective than the metric analyses reported before.

The standard deviation of the radius at the eminence of glabella in females (11.66) is about 3 times larger than in males (4.21). It was considered that in female, this place develops with much larger individual variation than in male, suggesting that females are more susceptible to misjudgment. In fact, as many as 5 in the 6 misclassified cases in the present series were females.

The tablet digitizer, though easy to operate as an input device, is associated with unavoidable reading errors that arise from the unequal interval of dots generated, the inaccurate manual tracing and the parallax produced by more or less inconstant viewpoint of the user. It is, therefore, desirable to improve the input procedure by, for instance, adopting such devices as a drum scanner.

\section{Acknowledgments}

We express our sincere gratitude to Prof. Mitani, H. and Drs. Kanamori, Y., Sugawara, J. and Soya, T., Department of Orthodontics, School of Dentistry, Tohoku.University, for valuable advice and offer of cephalograms, to Prof. Otani, S. and Dr. Miura, T., Department of Chemical Engineering, Faculty of Engineering, Tohoku University, for courteous advice of calculation process, and to Mr. Fukai, J., ditto, for the production of a program for the personal computer.

\section{References}

1) Ahlberg, J.H., Nilson, E.N. \& Walsh, J.L. (1967) The Theory of Splines and Their Applications. Academic press, New York, pp. 1-74.

2) Akamine, T. (1960) Forensic medical studies on sex differentiation in skulls. Shikoku Acta med., 16, Suppl. 1-21. (in Japanese with English summary)

3) Asai, Y. (1973) Growth changes of maxillofacial skeleton of Japanese from 12 to 20 years of age. J. Jap. orthod. Soc., 32, 61-98. (in Japanese with English summary)

4) Broadbent, B.H. (1931) A new x-ray technique and its application to orthodontics. Angle Orthodont., 1, 45-66.

5) Broadbent, B.H. (1937) The face of the normal child. Angle Orthodont., 17, 183208.

6) Fisher, R.A. (1936) The use of multiple measurements in taxonomic problems. Ann. Eugen., 7, 179-188.

7) Giles, E. \& Elliot, O. (1963) Sex determination by discriminant function analysis of crania. Amer. J. phys. Anthrop., 21, 53-68.

8) Graber, T.M. (1968) Orthodontics. 2nd ed., W.B. Saunders Co., Philadelphia, pp. 433-453.

9) Hanihara, K. (1958) Sexual diagnosis of Japanese long bones by means of discriminant function. $J$. anthrop. Soc. Nippon, 66, 187-196. (in Japanese with English abstract)

10) Hanihara, K. (1959) Sexual diagnosis of Japanese skulls and scapulae by means of discriminant function. J. anthrop. Soc. Nippon, 67, 192-197. (Japanese) 
11) Hanihara, K. (1981) Sexing of Japanese skeleton and teeth by discriminant function method. J. anthrop. Soc. Nippon, 89, 401-417. (in Japanese with English abstract)

12) Hanihara, K., Kimura, K. \& Minamidate, T. (1964) The sexing of Japanese skeleton by means of discriminant function. Jap. J. leg. Med., 18, 107-114. (in Japanese with English abstract)

13) Ichida, K. \& Yoshimoto, F. (1985) Spline Function and Its Application. Kyoikushuppan Co., Tokyo, pp. 26-27, 80-103, 114-121. (Japanese)

14) Kimura, T. (1971) Sex determination on the cross-section of human lower leg bones. Jap. J. leg. Med., 25, pp. 431-438. (in Japanese with English abstract)

15) Martin, R. (1914) Lehrbuch der Antheropologie, Verlag von Gustav Fischer, Jena, pp. 504-574.

16) Martin, R. \& Saller, K. (1957) Lehrbuch der Antheropologie, dritte Aufl., Gustav Fischer Verlag, Stuttgart.

17) Nanda, R.S. (1955) The rates of growth of several facial components measured from serial cephalometric roentgenograms. Amer. J. Orthodont., 41, 658-673.

18) Nakagawa, T. \& Koyanagi, Y. (1984) Experimental Data Analyses by Means of Least Square Method. Up applied mathematics series 7, 3rd ed., Tokyodaigakushuppankai, Tokyo, pp. 29-40. (Japanese)

19) Powell, M.J.D. (1970) Curve fitting by spline in one variable. In: Numerical Approximation to Functions and Data, edited by J.G. Hayes, Athione Press, London, pp. 65-83.

20) Ricketts, R.H. (1957) Planning treatment on the basis of the facial pattern and an estimate of its growth. Angle Orthodont., 27, 14-37.

21) Sassouni, V. (1958) Diagnosis and treatment planning via reontgenographic cephalometry. Amer. J. Orthodont., 44, 433-463.

22) Tanaka, T., Hanihara, K. \& Koizumi, K. (1979) Sex determination of the modern Japanese skull by means of discriminant function. Sapporo med. J., 48, 582-593. (in Japanese with English abstract) 